\title{
Nonequilibrium Effect in Ferromagnet-Insulator-Superconductor Tunneling Junction Currents
}

\author{
Michihide Kitamura, Kazuhiro Yamaki, Akinobu Irie \\ Department of Electrical and Electronic System Engineering, Utsunomiya University, Utsunomiya, Japan \\ Email:kitamura@cc.utsunomiya-u.ac.jp, kyamaki@cc.utsunomiya-u.ac.jp, iriea@cc.utsunomiya-u.ac.jp
}

Received 8 June 2016; accepted 22 July 2016; published 25 July 2016

Copyright (C) 2016 by authors and Scientific Research Publishing Inc. This work is licensed under the Creative Commons Attribution International License (CC BY). http://creativecommons.org/licenses/by/4.0/ c) (7) Open Access

\begin{abstract}
Nonequilibrium effect due to the imbalance in the number of the $\uparrow$ and $\downarrow$ spin electrons has been studied for the tunneling currents in the ferromagnet-insulator-superconductor (FIS) tunneling junctions within a phenomenological manner. It has been stated how the nonequilibrium effect should be observed in the spin-polarized quasiparticle tunneling currents, and pointed out that the detectable nonequilibrium effect could be found in the FIS tunneling junction at $77 \mathrm{~K}$ using $\mathrm{HgBa}_{2} \mathrm{Ca}_{2} \mathrm{Cu}_{3} \mathrm{O}_{8+\delta}(\mathrm{Hg}-1223)$ high- $T_{c}$ superconductor rather than $\mathrm{Bi}_{2} \mathrm{Sr}_{2} \mathrm{CaCu}_{2} \mathrm{O}_{8+\delta}(\mathrm{Bi}-2212)$ one.
\end{abstract}

\section{Keywords}

Nonequilibrium Effect, Ferromagnet-Insulator-Superconductor Tunneling Junction, Hg-1223, Bi-2212, Spin-Polarized Quasiparticle Tunneling

\section{Introduction}

Transition from an equilibrium to non-equilibrium state due to an external perturbation makes an output. The well known case is the transport phenomena, which can be understood by solving the Boltzmann equation for classical treatment and the Liouville equation for quantum one. Even in superconductors, the departure from the equilibrium state of the distribution function is found when the superconductors are set in the time and/or spatial modulations as an external perturbation. Such a situation, the nonequilibrium superconductivity, can be understood as a change of superconducting parameters induced by modifications of the distribution function of quasiparticle excitations. Studies for the nonequilibrium superconductivity have focused on the effects of not only the simple quasiparticle injection and extraction but also the spin-polarized quasiparticle transport. The valuable considerations have already been done by Tinkham [1]. In the case of simple quasiparticles, the phenomena can 
be described by introducing two parameters $T^{*}$ and $Q^{*}$ which represent the nonequilibrium temperature and quasiparticle charge density, respectively. In the case of the injection of spin-polarized quasiparticles, such as the quasiparticle tunneling in the ferromagnet-insulator-superconductor (FIS) tunneling junction, one can experimentally see the suppression of superconductivity whose origin is regarded as a pair-breaking mechanism of a Cooper-pair (CP).

CalTech group has extensively studied the nonequilibrium superconductivity under spin-polarized quasiparticle currents in the FIS tunneling junctions, and found that the phenomena manifesting nonequilibrium superconductivity in perovskite FIS heterostructure are observed and are attributed to the dynamic pair-breaking effect of spin-polarized quasiparticles in cuprate superconductors [2] [3]. We have experimentally studied the variation of the critical current $I_{c}$ of intrinsic Josephson junctions due to the spin injection and found that the observed modulation of $I_{c}$ of $\mathrm{Co} / \mathrm{Au} / \mathrm{Bi}_{2} \mathrm{Sr}_{2} \mathrm{CaCu}_{2} \mathrm{O}_{\mathrm{y}}$ mesa is attributed to the injection of the spin-polarized current [4] [5]. Recently, we have theoretically studied the charge and spin currents in FIS tunneling junction [6] and the spin flows in magnetic semiconductor-insulstor-superconductor (MS-I-S) tunneling junction [7] and found that the adopted MS-I-S tunneling junction seems to work as a switching device in which the spin up and down flows can be easily controlled by the external magnetic field [7].

Spintronics including not only the ferromagnets but also superconductors is one of the most attractive subjects in solid state physics and technology. Therefore, it is surely expected that such a research will grow rapidly. For example, Kaiser and Parkin have measured the tunneling spin polarization using a superconducting tunneling spectroscopy for $\mathrm{Al}_{2} \mathrm{O}_{3}$ tunnel barriers [8]. Rudenko et al. have observed the giant growth of the differential resistance using a tunnel junction consisting of superconducting lead with Heusler's ferromagnetic alloy $\mathrm{Co}_{2} \mathrm{CrAl}_{\text {, }}$ and pointed out that this effect is attributed to the appearance of a nonequilibrium state in the lead film as a result of spin injection into the superconductor [9].

Fundamental aspects of the proximity effect under nonequilibrium conditions even in normal metal-superconductor bilayers are not clear [10]. In the present paper, we phenomenologically study how the nonequilibrium effect due to spin injection should be observed in the spin-polarized quasiparticle tunneling along the $c$-axis of the FIS tunneling junctions. As a $F$ layer, a ferromagnetic $\mathrm{CrO}_{2}$ is selected because of its half metallic nature, i.e., a purely spin polarized, and $\mathrm{HgBa}_{2} \mathrm{Ca}_{2} \mathrm{Cu}_{3} \mathrm{O}_{8+\delta}(\mathrm{Hg}-1223)$ and $\mathrm{Bi}_{2} \mathrm{Sr}_{2} \mathrm{CaCu}_{2} \mathrm{O}_{8+\delta}(\mathrm{Bi}-2212)$ high- $T_{c}$ superconductors are adopted as a $\mathrm{S}$ layer. Hg-based superconducting cuprates form a series with the general formula $\mathrm{HgB}_{2} \mathrm{C}_{n-1} \mathrm{CunO}_{2 n+2+\delta}$ denoted as $\mathrm{Hg}-12 m n(m=n-1)$ with mainly $\mathrm{Ba}$ and $\mathrm{Ca}$ on the $\mathrm{B}$ and $\mathrm{C}$ sites, respectively. On increasing the number $\mathrm{n}$ of conducting $\mathrm{CuO}_{2}$ layers, the transition temperature $T_{c}$ progressively increases, reaching the maximum for Hg-1223 with a value of $135 \mathrm{~K}$, and then decreases. The amplitude $\Delta(0)$ at low temperature of the superconducting gap of $\mathrm{Hg}-1223$ is $75 \mathrm{meV}$ [11]. The structure of Bi-based superconducting cuprates form a series with the general formula $\mathrm{Bi}_{2} \mathrm{~B}_{2} \mathrm{C}_{n-1} \mathrm{CunO}_{2 n+4+\delta}$ denoted as Bi-22mn $(m=n-1)$ with mainly $\mathrm{Sr}$ and $\mathrm{Ca}$ on the $\mathrm{B}$ and $\mathrm{C}$ sites, respectively. The $T_{c}$ increases with an increasing number $n$ of $\mathrm{CuO}_{2}$ layers up to $110 \mathrm{~K}$ for $\mathrm{Bi}-2223$. The $T_{c}$ and $\Delta(0)$ of Bi-2212 we consider here are $86 \mathrm{~K}$ and $28 \mathrm{meV}$, respectively [11]. The crystal structures of Hg-1223 and Bi-2212 differ to each other, but there is a common feature such that these superconductors called "cuprate superconductors" include $\mathrm{CuO}_{2}$ layers showing a superconductive property. From the symmetry consideration for the $\mathrm{CuO}_{2}$ layer, these cuprate superconductors show the superconducting gap with $d_{x^{2}-y^{2}}$-symmetry so that the CPs are in a spin-singlet state.

It is considered for the present study that 1 ) the electron states in the vicinity of the Fermi level $E_{F}$ mainly come from $3 d$ orbitals of $\mathrm{Cu}$ and $\mathrm{Cr}$ atoms; 2) the density of states (DOS) that originated from the $3 d$ orbital shows a pointed structure meaning the localized nature, on the contrary to the DOS from $s$ and $p$ orbitals which show a broadened structure, i.e., the extended nature; therefore 3 ) the effective mass approximation, which is valid for the extended nature, may not be so good for the present system in which the electron states near the $E_{F}$ are fairly well localized; and 4) the size of the insulating layer $I$ is a realistic one, whose barrier strength is large enough, so it must be noted that 5) Blonder, Tinkham and Klapwijk (BTK) model [12] reaches to the tunneling Hamiltonian model since the probability of Andreev reflection decreases with the increasing the barrier strength of the $I$ layer. In the present paper, therefore, the tunneling Hamiltonian model based on the electrons with the Bloch states decided from the band structure calculations is adopted.

\section{Theoretical}

Tunneling current $i_{T, \sigma}^{(F)}(V)$ with a given spin $\sigma \quad(=\uparrow$ or $\downarrow)$ in the FIS tunneling junction is given as a function of an applied voltage $V$ as follows [6] [7]; 


$$
i_{T, \sigma}^{(F)}(V)=C \sum_{\mu_{S}} \sum_{L_{S}} \kappa_{\sigma}^{(F)}\left(\mu_{S}, L_{S}, V\right)
$$

Here note that the $S$ shown in Equation (1) is a symbol to identify the superconductor so that this symbol is used everywhere in the present paper. The charge and spin currents, $i_{T,+}^{(F)}(V)$ and $i_{T,-}^{(F)}(V)$, are calculated as

$$
i_{T, \pm}^{(F)}(V)=i_{T, \pm}^{(F)}(V)_{\text {expt }}=C \sum_{\mu_{S}} \sum_{L_{S}}\left\{\kappa_{\uparrow}^{(F)}\left(\mu_{S}, L_{S}, V\right) \pm \kappa_{\downarrow}^{(F)}\left(\mu_{S}, L_{S}, V\right)\right\} \equiv C i_{T, \pm}^{(F)}(V)_{c a l},
$$

where $C$ is a constant given by $(2 \pi e / \hbar) \tilde{T}^{2}$ and $\tilde{T}^{2} \equiv\left|T_{\uparrow}\right|^{2}+\left|T_{\downarrow}\right|^{2}$. In the present paper, we consider the nonequilibrium effect on the charge current $i_{T,+}^{(F)}(V)$. The $\kappa_{\sigma}^{(F)}\left(\mu_{S}, L_{S}, V\right)$ is defined as

$$
\kappa_{\sigma}^{(F)}\left(\mu_{S}, L_{S}, V\right)=\eta_{\sigma} \sum_{\mathbf{k}}^{\Omega_{S}} \Theta_{\sigma}^{(F)}\left(\xi_{\mathbf{k}}^{(S)}, \Delta_{\mathbf{k}}, e V\right)\left|\lambda_{L_{S}}^{\left(\mu_{S}\right)}(\mathbf{k})\right|^{2},
$$

where $\Omega_{S}$ is the first Brillouin zone of $S$. The $\lambda_{L_{S}}^{\left(\mu_{S}\right)}(\mathbf{k})$ is the coefficient in the expansion by the Bloch orbitals $\chi_{L_{S}}^{\left(\mu_{S}\right)}(\mathbf{k}, \mathbf{r})$ of the total wavefuntion $\Psi_{\mathbf{k}}(\mathbf{r})$ of $S$ such as

$$
\Psi_{\mathbf{k}}(\mathbf{r})=\sum_{\mu_{S}} \sum_{L_{S}} \lambda_{L_{S}}^{\left(\mu_{S}\right)}(\mathbf{k}) \chi_{L_{S}}^{\left(\mu_{S}\right)}(\mathbf{k}, \mathbf{r})
$$

where $\mu_{S}$ and $L_{S}$ are the site to be considered and the quantum state of atomic orbital of $S$, respectively.

The $\eta_{\sigma}$ in Equation (3) is the tunneling probability of a $\sigma$-spin electron in the FIS tunneling junction defined by

$$
\eta_{\sigma} \equiv \frac{\left|T_{\sigma}\right|^{2}}{\tilde{T}^{2}},
$$

so that the value of $\eta_{\sigma}$ strongly depends on the magnetic nature of an insulating layer $I$. As the $I$, we consider here the non-magnetic layer, thus the tunneling probabilities of majority $(\uparrow)$ and minority $(\downarrow)$ spin electrons must be equal each other, i.e., $\eta_{\uparrow}=\eta_{\downarrow}=1 / 2$.

As a tunneling process, coherent, incoherent and WKB cases can be considered. In the present paper, the incoherent tunneling is mainly studied. The reason is described later. In the incoherent tunneling case, the $\Theta_{\sigma}^{(F)}\left(\xi_{\mathbf{k}}^{(S)}, \Delta_{\mathbf{k}}, e V\right)$ in Equation (3) denoted as $\Theta_{\sigma}^{(F)}\left(\xi_{\mathbf{k}}^{(S)}, \Delta_{\mathbf{k}}, e V\right)_{\text {Inc }}$ is given by [6]

$$
\begin{aligned}
\Theta_{\sigma}^{(F)}\left(\xi_{\mathbf{k}}^{(S)}, \Delta_{\mathbf{k}}, e V\right)_{I n c}= & \left\{f\left(E_{\mathbf{k}}-e V\right)-f\left(E_{\mathbf{k}}\right)\right\} D_{\sigma}^{(F)}\left(E_{\mathbf{k}}-e V\right) \\
& +\left\{f\left(E_{\mathbf{k}}\right)-f\left(E_{\mathbf{k}}+e V\right)\right\} D_{\sigma}^{(F)}\left(-E_{\mathbf{k}}-e V\right)
\end{aligned}
$$

where $f$ is a Fermi-Dirac distribution function and $D_{\sigma}^{(F)}(\varepsilon)$ is the TDOS of the $F$ layer for $\sigma$ spin state as a function of energy $\varepsilon$. The $E_{\mathbf{k}}$ is a quasiparticle excitation energy defined by $\sqrt{\xi_{\mathbf{k}}^{2}+\Delta_{\mathbf{k}}^{2}}$, where the $\xi_{\mathbf{k}}$ is an one electron energy relative to the Fermi level $E_{F}$ and the $\Delta_{\mathbf{k}}$ is a superconducting energy gap given by $\Delta\left(T_{\text {samp }}\right) \cos 2 \theta_{\mathbf{k}}$ with a sample temperature $T_{\text {samp }}$.

The one electron energy $\xi_{\mathbf{k}}$ is calculated on the basis of the band theory using a universal tight-binding parameters (UTBP) method proposed by Harrison [13]. The energies of the atomic orbitals used in the band structure calculations have been calculated by using the spin-polarized self-consistent-field (SP-SCF) atomic structure calculations based on the Herman and Skillman prescription [14] using the Schwarz exchange correlation parameters [15].

\section{Results and Discussion}

First of all, we must check how the current-voltage $(I-V)$ characteristics are changed due to the change of tunneling mechanism such as coherent, incoherent and WKB ones. In order to do so, we have calculated the $I-V$ characteristics $i_{T,+}^{(F)}(V)_{c a l}$ of the FIS tunneling junction for these three cases, where the $F$ is the ferromagnetic $\mathrm{CrO}_{2}$ with a half metal phase, the $I$ is a nonmagnetic insulating layer with a real dimensional size, and the $S$ is the Hg-1223 high- $T_{c}$ superconductor. Here we wish to emphasize that the numerical calculations for the coherent and WKB cases need a very large CPU time as compared with the incoherent case [6]. The $i_{T,+}^{(F)}(V)_{c a l}$ 's at $T_{\text {samp }}$ 
$=4.2 \mathrm{~K}$ calculated for above three cases have told us that 1 ) the result calculated for the coherent case shows a unrealistic behavior so that there are some regions in which the differential conductance $\mathrm{d} I / \mathrm{d} V$ is calculated as a negative value, 2) that for the incoherent case is reasonable, and 3) that for the WKB case is fairly similar to that for the incoherent one, but there are regions in which the $\mathrm{d} I / \mathrm{d} V$ is calculated as a negative value. From above, we consider here only the incoherent tunneling case.

Next, we must consider the effect of the external magnetic field. In the present paper, the FIS tunneling junction, in which the $F$-layer shows a magnetization because of a half metallic $\mathrm{CrO}_{2}$ so that the spin-polarized quasiparticle injection has been well done for no applied field, is considered. Generally, the magnetic field has an obvious effect on the transition temperature $T_{c}$ and superconducting gap $\Delta$, however, the external magnetic field we consider here is a field made by the magnetization of the $\mathrm{CrO}_{2}$-layer. Therefore, it seems that the effect of the external magnetic field may be small. In the present paper, thus, its effect has been taken into account by using the same method done by Tedrow and Meservey [16]. Namely, the quasiparticle excitation energy $E_{\mathbf{k}}$ has been replaced by $E_{\mathbf{k}} \mp \mu_{B} H$ for the majority and the minority spin, respectively. Actually, we did a calculation for the external magnetic induction $B_{\text {ext }}$ with the value of $1 \mathrm{~T}$, and found that there is no detectable difference between the calculations for $B_{e x t}=0$ and $1 \mathrm{~T}$. In the present paper, therefore, the effect of the external magnetic field has not been considered anymore. In the following, therefore, we consider only the incoherent tunneling case with no external magnetic field.

Experimental current $i_{T, \pm}^{(F)}(V)_{\text {expt }}$ is proportional to the calculated one $i_{T, \pm}^{(F)}(V)_{c a l}$. Therefore, if the logarithmic derivative is taken for both currents, then a following relation is held

$$
\frac{\mathrm{d}}{\mathrm{d} V} \log i_{T, \pm}^{(F)}(V)_{\text {expt }}=\frac{\mathrm{d}}{\mathrm{d} V} \log i_{T, \pm}^{(F)}(V)_{\text {cal }} \equiv L D .
$$

This relation clearly shows that the logarithmic derivative $L D$ calculated by using numerically calculated values is exactly equal to that by using the experimental one. In the following, therefore, we show only the $L D$ values deduced from the full numerically calculated charge current $i_{T,+}^{(F)}(V)_{c a l}$.

In the FIS tunneling junction, it is easily supposed that the imbalance in the number of the $\uparrow$ and $\downarrow$ spin electrons makes a decrease in the number of CPs. This is just a nonequilibrium effect that we consider here. The decrease in the number of CPs makes a decrease in the amplitude $\Delta(\tau)$ of the superconducting gap, where $\tau \equiv T / T_{c}$. Therefore, in order to take into account the influence of such a nonequilibrium effect, we introduce a parameter $\gamma(\tau)$ with a value between 0 and 1 , by which the $\Delta(\tau)$ is reduced to $(1-\gamma(\tau)) \Delta(\tau)$. Here note that $1-\gamma(\tau)$ is equal to the parameter $\zeta$ we have introduced previously [7]. It is clear that the condition $\gamma(\tau)=0$ means the no consideration for the nonequilibrium effect due to the imbalance in the number of the $\uparrow$ and $\downarrow$ spin electrons. The parameter $\gamma(\tau)$ directly reflects the imbalance in spin population, so it must be noted that the $\gamma(\tau)$ should be treated separately apart from the parameter $T^{*}$ which represents the nonequilibrium temperature. At low temperature region $\tau \simeq 0$, the nonequilibrium effect we consider here should be small and its temperature variation may also be small because of a huge number of CPs at the low temperature region. Therefore, it may be reasonable to suppose that $\gamma(0)=0$ and $\gamma^{\prime}(0)=0$. For the $\gamma(\tau)$, thus, we a priori assume that

$$
\gamma(\tau)=\gamma(1) \tau^{2}
$$

where $\gamma(1)$ is an adjustable parameter with $0 \leq \gamma(1) \leq 1$. Equation (8) is just a phenomenological.

The differences $L D_{\text {FIS }}-L D_{\text {NIS }}$ of the logarithmic derivatives $L D_{F I S}$ and $L D_{\text {NIS }}$ deduced from the charge currents $i_{T,+}^{(F)}(V)_{c a l}$ calculated for the FIS and NIS tunneling junctions are shown in Figures 1(a)-(f). Figure 1(a), Figure 1(b) and Figure 1(c) are results obtained by using a ferromagnetic half metal $\mathrm{CrO}_{2}$ as $F$, an $\mathrm{Al}$ metal as $N$ and a $\mathrm{HgBa}_{2} \mathrm{Ca}_{2} \mathrm{Cu}_{3} \mathrm{O}_{8+\delta}$ (Hg-1223) high- $T_{c}$ superconductor as $S$, and (d), (e) and (f) are those by using the $\mathrm{CrO}_{2}$ as $\mathrm{F}$, the $\mathrm{Al}$ as $\mathrm{N}$ and a $\mathrm{Bi}_{2} \mathrm{Sr}_{2} \mathrm{CaCu}_{2} \mathrm{O}_{8+\delta}(\mathrm{Bi}-2212)$ high- $T_{c}$ superconductor as $\mathrm{S}$. As already stated, the $T_{c}$ of $\mathrm{Hg}-1223$ and $\mathrm{Bi}-2212$ is 135 and $86 \mathrm{~K}$, and the $\Delta(0)$ of those are 75 and $28 \mathrm{meV}$, respectively [11]. In order to make a comparison with the experiment, it is important to define the sample temperature $T_{\text {samp }}$ even in the theoretical studies. In the present calculations, therefore, the reduced sample temperature $\tau_{\text {samp }}\left(\equiv T_{\text {samp }} / T_{c}\right)$ has been selected as 0.1 for Figure 1(a) and Figure 1(d), 0.5 for Figure 1(b) and Figure 1(e), and 0.9 for Figure 1 (c) and Figure 1(f), tentatively. Therefore, for Figures $1(\mathrm{a})$-(f), the realistic sample temperature $T_{\text {samp }}$ is 13.5 , 67.5, 121.5, 8.6, 43.0 and $77.4 \mathrm{~K}$, and the resultant $\Delta\left(T_{\text {samp }}\right)$ is 75, 72, 40, 28, 27 and $15 \mathrm{meV}$, respectively. The horizontal axis is the normalized voltage $V_{N}$ defined by $V / \Delta\left(T_{\text {samp }}\right)$ and the vertical one is the LD in unit 

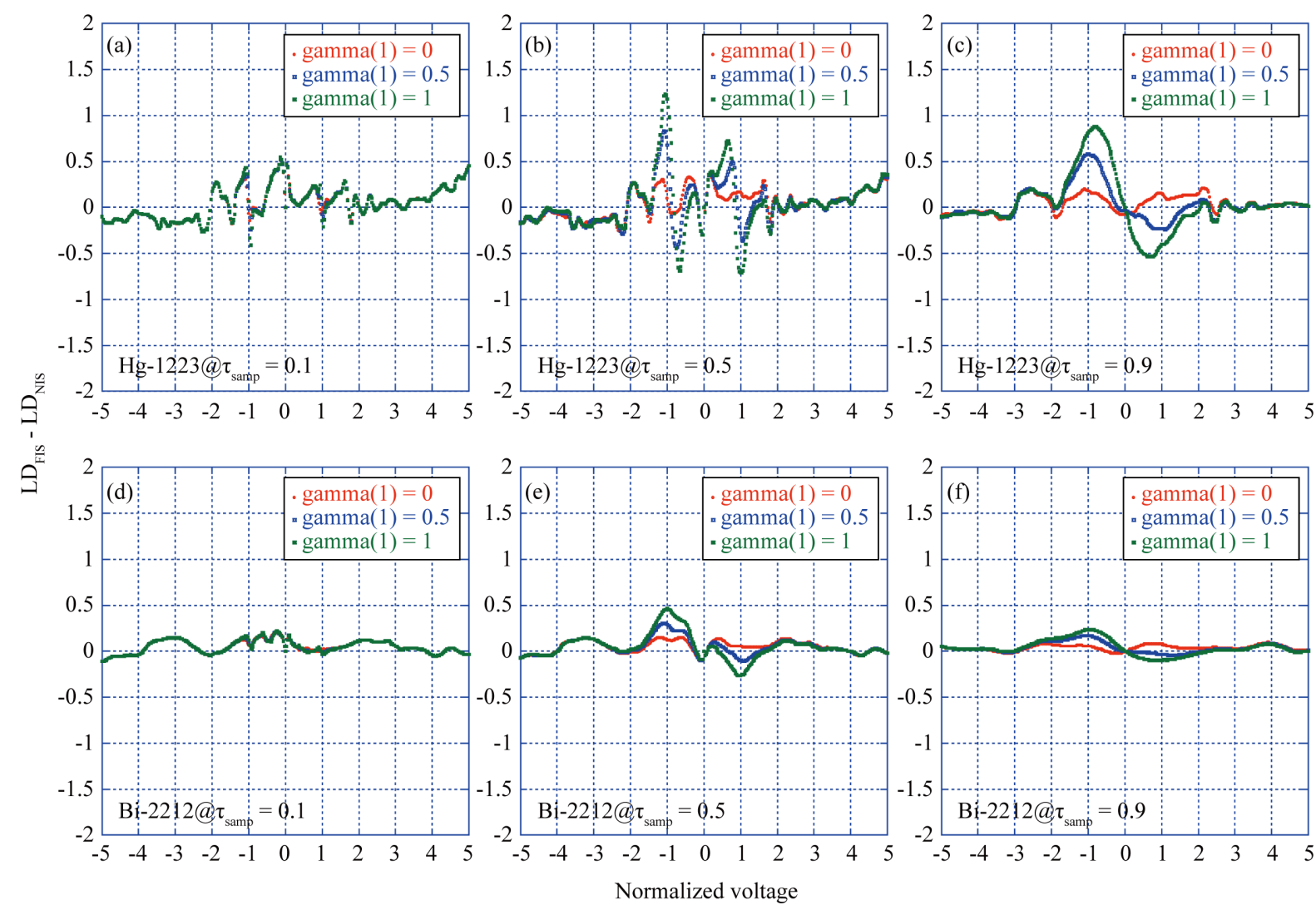

Figure 1. Plots of the difference $L D_{\text {FIS }}-L D_{\text {NIS }}$ of the logarithmic derivatives $L D_{F I S}$ and $L D_{N I S}$. (a), (b) and (c) are results obtained by using the FIS and NIS tunneling junctions with a ferromagnetic half metal $\mathrm{CrO}_{2}$ as $F$, an $\mathrm{Al}$ metal as $N$ and a $\mathrm{HgBa}_{2} \mathrm{Ca}_{2} \mathrm{Cu}_{3} \mathrm{O}_{8+\delta}$ (Hg-1223) high- $T_{c}$ superconductor as $S$, and (d), (e) and (f) are those with the $\mathrm{CrO}_{2}$ as $F$, the $\mathrm{Al}$ as $N$ and a $\mathrm{Bi}_{2} \mathrm{Sr}_{2} \mathrm{CaCu}_{2} \mathrm{O}_{8+\delta}(\mathrm{Bi}-2212)$ high- $T_{C}$ superconductor as $S$. The $T_{c}$ is 135 and $86 \mathrm{~K}$ and the amplitude $\Delta(0)$ at low temperature is 75 and $28 \mathrm{meV}$, respectively, for the $\mathrm{Hg}-1223$ and $\mathrm{Bi}-2212$ high- $T_{c}$ superconductors [11]. The horizontal axis is the normalized voltage $V_{N}$ defined by $V / \Delta\left(T_{\text {samp }}\right)$ and the vertical one is the $L D$ in unit of $1 / V_{N}$. The reduced sample temperature $\tau_{\text {samp }}\left(\equiv T_{\text {samp }} / T_{c}\right)$ for (a) and (d) is 0.1, that for (b) and (e) is 0.5 and that for (c) and (f) is 0.9. Therefore, for (a), (b), (c), (d), (e) and (f), the sample temperature $T_{\text {samp }}$ is $13.5,67.5,121.5,8.6,43.0$ and $77.4 \mathrm{~K}$, and the $\Delta\left(T_{\text {samp }}\right)$ is $75,72,40,28,27$ and 15 meV, respectively. The $\gamma(1)$ with the values of $0,0.5$ and 1 has been selected and the corresponding curves have been drawn by red, blue and green colors, respectively.

of $1 / V_{N}$. The numerical calculations have been done at no external magnetic field and a voltage interval $-5 \leq$ $V_{N} \leq 5$, so it must be noted that the real voltage $V=V_{N} \Delta\left(T_{\text {samp }}\right)$ differs for all. The $\gamma(1)$ with the values of 0 , 0.5 and 1 has been selected tentatively, and the corresponding curves have been drawn by red, blue and green colors, respectively. The condition $\gamma(1)=0$ means the no consideration for the nonequilibrium effect due to the imbalance in the number of the $\uparrow$ and $\downarrow$ spin electrons, so it is clear that (1) the structures found in curves drawn by red color with $\gamma(1)=0$ are caused to the difference between the densities of states of a ferromagnetic half-metal $\mathrm{Cr}$ and a normal simple metal Al and (2) the change of curves due to the increase of $\gamma(1)$ directly shows the nonequilibrium effect due to the imbalance of the spin population. The calculations show that (1) the nonequilibrium effect is found at $\tau_{\text {samp }}=0.5$ and 0.9 , and (2) the remarkable change is found in Hg-1223 high- $T_{c}$ superconductor rather than $\mathrm{Bi}-2212$ one. This is caused to the fact that the $\Delta(0)$ of Hg-1223 superconductor is fairly larger than that of Bi-2212 one. We have a priori assumed that the phenomenological parameter $\gamma$ can be regarded as a function of only the $\tau$. However, there is a fact that the CP becomes much stable due to the increase of the superconducting gap, and there are some theoretical studies for the superconducting gap such that the spin-exchange interaction could be considered as a one of the origins of the attractive interaction for $\mathrm{CP}$ [17]-[23]. Therefore, it may be reasonable to suppose that the $\gamma$ should also be correlated with the superconducting gap $\Delta$, that is, $\gamma$ may be a function such as $\gamma(\tau, \Delta)$. Only the experimental study can clarify this conjecture. 
In order to make a comparison with the experimental study, we have chosen two temperatures 4.2 and $77 \mathrm{~K}$ as

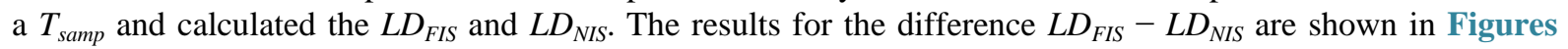
2(a)-(d). Figure 2(a) and Figure 2(b) are results obtained by using FIS and NIS tunneling junctions with a $\mathrm{CrO}_{2}$ as $F$, an $\mathrm{Al}$ metal as $N$ and a $\mathrm{HgBa}_{2} \mathrm{Ca}_{2} \mathrm{Cu}_{3} \mathrm{O}_{8+\delta}(\mathrm{Hg}-1223)$ high- $T_{c}$ superconductor as $S$, and (c) and (d) are those with the $\mathrm{CrO}_{2}$ as $F$, the $\mathrm{Al}$ as $N$ and a $\mathrm{Bi}_{2} \mathrm{Sr}_{2} \mathrm{CaCu}_{2} \mathrm{O}_{8+\delta}(\mathrm{Bi}-2212)$ high- $T_{c}$ superconductor as $S$. The $T_{\text {samp }}$ is $4.2 \mathrm{~K}$ for (a) and (c) and $77 \mathrm{~K}$ for (b) and (d) and the $\Delta(4.2)$ and $\Delta(77)$ of Hg-1223 are 75 and $69.7 \mathrm{meV}$, and those of Bi-2212 are 28 and $15.2 \mathrm{meV}$, respectively. The horizontal axis of Figure 2 is in the real voltage, so it should be emphasized that the calculated results can be directly compared with the experimental ones. Figure 2 shows that (1) the nonequilibrium effect due to the imbalance in the number of the $\uparrow$ and $\downarrow$ spin electrons is not found at $4.2 \mathrm{~K}$ as is shown in (a) and (c), but is found at $77 \mathrm{~K}$ in (b) and (d), and (2) its effect is clearly found in (b), that is, the case of the FIS tunneling junction using Hg-1223 superconductor at $77 \mathrm{~K}$.

At the high voltage region, the I-V curve of FIS tunneling junction approaches to the ohmic line. Namely, the effect of the variation of superconducting gap decreases with an increasing the voltage applied to the junction.

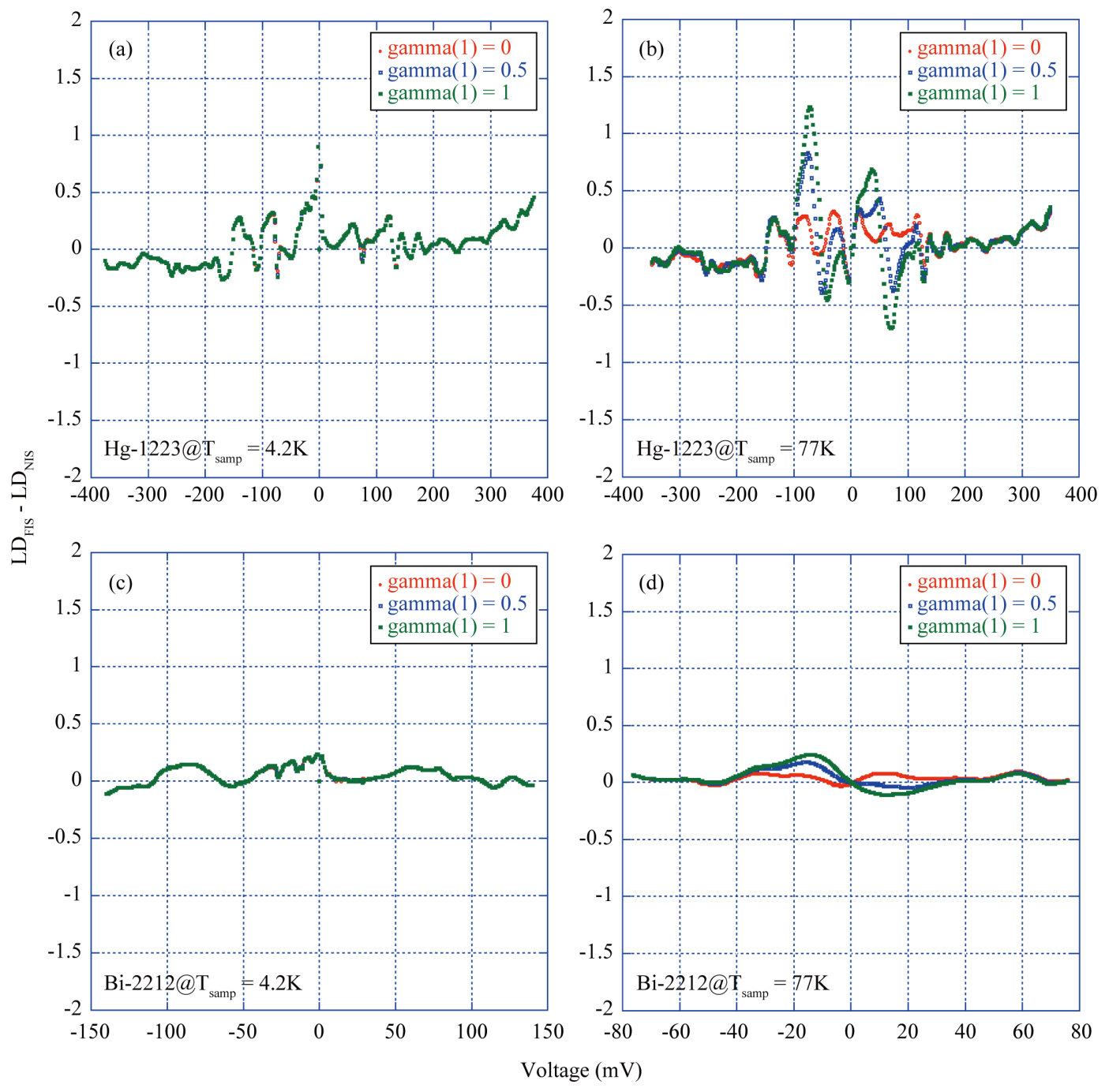

Figure 2. Basically the same as in Figure 1 but for the case in which $T_{\text {samp }}$ has been set to $4.2 \mathrm{~K}$ for (a) and (c) and $77 \mathrm{~K}$ for (b) and (d), and the horizontal axis has been given by the real voltage $(\mathrm{mV})$, in order to make a comparison with the experimental study. (a) and (b) are results obtained by using a $\mathrm{CrO}_{2}$ as $F$, an $\mathrm{Al}$ metal as $N$ and a $\mathrm{HgBa}_{2} \mathrm{Ca}_{2} \mathrm{Cu}_{3} \mathrm{O}_{8+\delta}(\mathrm{Hg}-1223)$ high- $T_{c}$ superconductor as $S$, and (c) and (d) are those by using the $\mathrm{CrO}_{2}$ as $F$, the $\mathrm{Al}$ as $N$ and a $\mathrm{Bi}_{2} \mathrm{Sr}_{2} \mathrm{CaCu}_{2} \mathrm{O}_{8+\delta}(\mathrm{Bi}-2212)$ high $T_{c}$ superconductor as $S$. The $\Delta(4.2)$ and $\Delta(77)$ of $\mathrm{Hg}-1223$ are 75 and $69.7 \mathrm{meV}$, and those of $\mathrm{Bi}-2212$ are 28 and $15.2 \mathrm{meV}$, respectively. 
This is a reason why the value of $L D_{F I S}-L D_{\text {NIS }}$ at the high voltage region remains the same for the change of the $\gamma(1)$, as is shown in Figure 1 and Figure 2.

Our phenomenological approach for the nonequilibrium effect states that if the experiments for the difference $L D_{F I S}-L D_{\text {NIS }}$ using the $\mathrm{CrO}_{2}$ as $F$, the $\mathrm{Al}$ as $N$ and the $\mathrm{Hg}-1223$ high- $T_{C}$ superconductor as $S$ are done at two temperatures such as 4.2 and $77 \mathrm{~K}$ and the detectable differences are found at these temperatures, then such a difference is directly correlated with the nonequilibrium effect due to the imbalance in the number of the $\uparrow$ and $\downarrow$ spin electrons.

\section{Summary}

For the $c$-axis tunneling currents observed in the ferromagnet-insulator-superconductor (FIS) tunneling junctions, we have phenomenologically studied the nonequilibrium effect due to the imbalance in the number of the $\uparrow$ and $\downarrow$ spin electrons, in order to see how the nonequilibrium effect due to spin injection should be observed in the spin-polarized quasiparticle tunneling. We have showed that 1 ) the nonequilibrium effect is found at $77 \mathrm{~K}$ rather than $4.2 \mathrm{~K}$, and 2) its effect is clearly found in the FIS tunneling junction using the Hg-1223 high- $T_{c}$ superconductor rather than $\mathrm{Bi}-2212$ one as $S$.

\section{References}

[1] Tinkham, M. (1996) Introduction to Superconductivity. 2nd Edition, McGraw-Hill, New York.

[2] Yeh, N.-C., Vasquez, R.P., Fu, C.C., Samoilov, A.V., Li, Y. and Vakili, K. (1999) Nonequilibrium Superconductivity Under Spin-Polarized Quasiparticle Currents in Perovskite Ferromagnet-Insulator-Superconductor Heterostructures. Physical Review B, 60, Article ID: 10522.

[3] Fu, C.C., Huang, Z. and Yeh, N.-C. (2002) Spin-Polarized Quasiparticle Transport in Cuprate Superconductors. Physical Review B, 65, Article ID: 224516.

[4] Irie, A., Arakawa, N., Sakuma, H., Kitamura, M. and Oya, G. (2010) Magnetization-Dependent Critical Current of Intrinsic Josephson Junctions in $\mathrm{Co} / \mathrm{Au} / \mathrm{Bi}_{2} \mathrm{Sr}_{2} \mathrm{CaCu}_{2} \mathrm{O}_{\mathrm{y}}$ Mesa Structures. Journal of Physics: Conference Series, 234, Article ID: 042015. http://dx.doi.org/10.1088/1742-6596/234/4/042015

[5] Irie, A., Arakawa, N., Kitamura, M., Sakuma, H. and Oya, G. (2011) Control of Critical Current of Intrinsic Josephson Junctions Due to Spin Injection. IEEE Transactions on Applied Superconductivity, 21, 741-44. http://dx.doi.org/10.1109/TASC.2010.2090632

[6] Kitamura, M., Uchiumi, Y. and Irie, A. (2014) Charge and Spin Currents in Ferromagnet-Insulator-Superconductor Tunneling Junctions Using Hg-1223 High- $\mathrm{T}_{\mathrm{c}}$ Superconductor. International Journal of Superconductivity, 2014, Article ID: 957045. http://dx.doi.org/10.1155/2014/957045

[7] Kitamura, M. and Irie, A. (2015) Spin Flows in Magnetic Semiconductor/Insulator/Superconductor Tunneling Junction. International Journal of Superconductivity, 2015, Article ID: 273570. http://dx.doi.org/10.1155/2015/273570

[8] Kaiser, C. and Parkin, S.S. (2004) Spin Polarization in Ferromagnet/Insulator/Superconductor Structures with the Superconductor on Top of the Barrier. Applied Physics Letters, 84, 3582. http://dx.doi.org/10.1063/1.1737485

[9] Rudenko, É.M., Korotash, I.V., Kudryavtsev, Yu.V., Krakavny̌̆, A.A., Belogolovskiı̌, M.A. and Boĭlo, I.V. (2010) Spin Injection and Giant Tunnel-Current Blocking in Ferromagnet-Superconductor Heterostructures. Low Temperature Physics, 36, 186. http://dx.doi.org/10.1063/1.3314258

[10] Belogolovskii, M. (2015) Applied Superconductivity, Handbook on Devices and Applications. Wiley, New York.

[11] Poole Jr, C.P. (2000) Handbook of Superconductivity. Academic Press, San Diego.

[12] Blonder, G.E., Tinkham, M. and Klapwijk, T.M. (1982) Transition from Metallic to Tunneling Regimes in Superconducting Microconstrictions: Excess Current, Charge Imbalance, and Supercurrent Conversion. Physical Review B, 25, 4515. http://dx.doi.org/10.1103/PhysRevB.25.4515

[13] Harrison, W.A. (1999) Elementary Electronic Structure. World Scientific, Singapore.

[14] Herman, F. and Skillman, S. (1999) Atomic Structure Calculations. Prentice-Hall, Englewood Cliffs.

[15] Schwarz, K. (1972) Optimization of the Statistical Exchange Parameter $\alpha$ for the Free Atoms H through Nb. Physical Review B, 5, 2466. http://dx.doi.org/10.1103/PhysRevB.5.2466

[16] Tedrow, P.M. and Meservey, R. (1971) Spin-Dependent Tunneling into Ferromagnetic Nickel. Physical Review Letters, 26, 192-195. http://dx.doi.org/10.1103/PhysRevLett.26.192

[17] Ohkawa, F.J. (1987) Copper Pairs of $d \gamma$-Symmetry in Simple Square Lattices. Journal of the Physical Society of Japan, 56, 2267-2270. http://dx.doi.org/10.1143/JPSJ.56.2267 
[18] Tanamoto, T., Kohno, H. and Fukuyama, H. (1992) Fermi Surface and Spin Fluctuations in Extended $t-J$ Model. Journal of the Physical Society of Japan, 61, 1886-1890. http://dx.doi.org/10.1143/JPSJ.61.1886

[19] Lavanga, M. and Stemmann, G. (1994) Spin Excitations of Two-Dimensional-Lattice Electrons: Discussion of Neutron-Scattering and NMR Experiments in High-Tc Superconductors. Physical Review B, 49, 4235-4250. http://dx.doi.org/10.1103/PhysRevB.49.4235

[20] Pao, C.-H. and Bickers, N.E. (1994) Anisotropic Superconductivity in the 2D Hubbard Model: Gap Function and Interaction Weight. Physical Review Letters, 72, 1870-1873. http://dx.doi.org/10.1103/PhysRevLett.72.1870

[21] Monthoux, P. and Scalapino, D.J. (1994) Self-Consistent $\mathrm{dx}^{2}-\mathrm{y}^{2}$ Pairing in a Two-Dimensional Hubbard Model. Physical Review Letters, 72, 1874-1877. http://dx.doi.org/10.1103/PhysRevLett.72.1874

[22] Maki, K. and Won, H. (1994) Spin Fluctuations of D-Wave Superconductors. Physical Review Letters, 72, 1758. http://dx.doi.org/10.1103/PhysRevLett.72.1758

[23] Kitamura, M., Irie, A. and Oya, G. (2005) Phenomenological Approach to the Superconducting $\mathrm{Gap}$ of $\mathrm{Bi}_{2} \mathrm{Sr}_{2} \mathrm{CaCu}_{2} \mathrm{O}_{8+\delta}$. Physica C: Superconductivity and Its Applications, 423, 190-198. http://dx.doi.org/10.1016/j.physc.2005.04.015

\section{Submit or recommend next manuscript to SCIRP and we will provide best service for you:}

Accepting pre-submission inquiries through Email, Facebook, LinkedIn, Twitter, etc.

A wide selection of journals (inclusive of 9 subjects, more than 200 journals)

Providing 24-hour high-quality service

User-friendly online submission system

Fair and swift peer-review system

Efficient typesetting and proofreading procedure

Display of the result of downloads and visits, as well as the number of cited articles

Maximum dissemination of your research work

Submit your manuscript at: http://papersubmission.scirp.org/ 